\title{
Atividade Ovariana em Fêmeas Bovinas da Raça Holandesa e Mestiças Holandês x Zebu, Durante Dois Ciclos Estrais Normais Consecutivos ${ }^{1}$
}

\author{
Nadja Gomes Alves ${ }^{2}$, Eduardo Paulino da Costa ${ }^{3}$, José Domingos Guimarães ${ }^{3}$, \\ Márcio Ribeiro Silva ${ }^{4}$, Belmiro Zamperlini ${ }^{5}$, Flávio Marcos Juqueira Costa ${ }^{4}$, \\ Anselmo Domingos Ferreira Santos ${ }^{2}$, Tamires Miranda Neto ${ }^{4}$
}

\begin{abstract}
RESUMO - Foram utilizadas 25 fêmeas, distribuídas de acordo com o grau de sangue Holandês x Zebu nos grupos: $\mathrm{I}=1 / 2$ Holandês x Zebu; II = 3/4 Holandês x Zebu; III= 15/16 Holandês x Zebu; IV= Holandês-PB-PC; e V=7/8 Holandês x Zebu. Após o segundo estro natural pós-parto, esses animais foram monitorados por exames ultra-sonográficos diários por dois ciclos estrais consecutivos, para verificar a atividade ovariana quanto à dinâmica folicular e à atividade do corpo lúteo. O período interovulatório

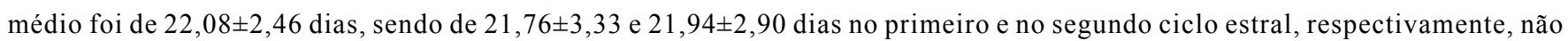
havendo diferença entre os grupos estudados. Observou-se o crescimento de uma (6\%), duas (78\%) e três (16\%) ondas foliculares durante o ciclo estral, com a predominância de duas ondas foliculares. O período interovulatório dos ciclos com três ondas mostrou-se

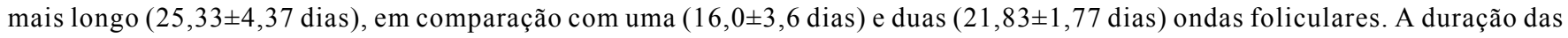
ondas foliculares para ciclos estrais com três ondas de crescimento folicular foi de $11,0 \pm 3,69 ; 7,17 \pm 1,60$; e $8,5 \pm 1,52$ dias na $1^{\text {a }}$,

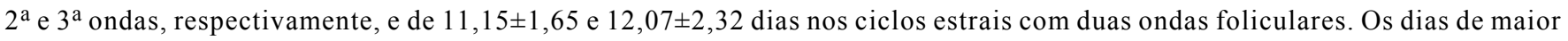
concentração plasmática de progesterona no $1^{\circ}$ e $2^{\circ}$ ciclos estrais foram, respectivamente, de $13,6 \pm 3,0(7,29 \pm 4,3 \mathrm{ng} / \mathrm{mL})$ e $11,8 \pm 3,4$ $(5,6 \pm 2,0 \mathrm{ng} / \mathrm{mL})$ dias, não se mostrando diferentes entre os grupamentos genéticos e ciclos estrais estudados. Pelas análises dos dados, pode-se concluir que os diferentes grupamentos genéticos apresentaram o mesmo comportamento na dinâmica folicular e que as concentrações de progesterona, embora diferentes em números absolutos entre os grupamentos, mostraram-se indicativas de atividade luteal normal.
\end{abstract}

Palavras-chave: bovino, corpo lúteo, dinâmica folicular

\section{Ovarian Activity in Holstein and Crossbreed Holstein x Zebu Cows During Two Normal Estrous Cycles}

\begin{abstract}
To verify the ovarian activity in respect to follicular dynamics and corpus luteum activity, 25 cows distributed in five experimental groups according to the crossbreeding level as: group 1: 1/2 Holstein x Zebu, group 2: $3 / 4$ Holstein x Zebu, group 3: 15/16 Holstein x Zebu, group 4: crossbreed pure Holstein and group 5: 7/8 Holstein x Zebu, were daily monitored by ultrasonography examination during two estrous cycle after the second postpartum cycle. The average in days for the interovulatory period were respectively, for the first and second cycles: $21.76 \pm 3.33$ and $21.94 \pm 2.90$. There was no difference between these values. The average for the interovulatory period between cycles was $22,08 \pm 2,46$ days. In this experiment, the development of one (16\%), two (78\%) and three (16\%) follicular waves was verified, with predominance of two follicular waves. The interovulatory period in three waves cycles ( $25.33 \pm 4.36$ days) was longer when compared to one (16.0 \pm 3.6 days) and two $(21.69 \pm 1.97$ days) waves cycles. The follicular waves average length were: $11.0 \pm 3.69,7.17 \pm 1.60$ and $8.5 \pm 1.52$ days, respectively, for the first, second and third follicular waves. In estrous cycles where only two waves occurred, the waves average length were: $11.15 \pm 1.65$ and $12.07 \pm 2.82$ days. The highest progesterone concentrations in the first and second estrous cycle were, respectively, in the $13.6 \pm 3.0(7.29 \pm 4.3 \mathrm{ng} / \mathrm{mL})$ and $11.8 \pm 3.4(5.6 \pm 2.0 \mathrm{ng} / \mathrm{mL})$ days and were not different among experimental groups and estrous cycle. After the analyses, it was concluded that the different experimental groups presented the same follicular dynamics behavior, and despite the differences in absolute numbers in progesterone concentration among the groups, they were indicative of normal luteal activity.
\end{abstract}

Key Words: bovine, corpus luteum, follicular dynamics

\footnotetext{
${ }^{1}$ Apoio financeiro da FAPEMIG - Fundação de Amparo à Pesquisa do Estado de Minas Gerais.

${ }^{2}$ Aluno de Doutorado - Universidade Federal de Viçosa - Departamento de Zootecnia. Avenida P. H. Rolfs, s/n - Campus Universitário Viçosa - MG - CEP: 36571-000. E.mail: nadja10@globo.com

3 Universidade Federal de Viçosa - Departamento de Veterinária. E.mail: epcosta@mail.ufv.br

${ }^{4}$ Médico-Veterinário.

${ }^{5}$ Universidade Federal de Viçosa - Departamento de Zootecnia.
} 


\section{Introdução}

A pecuária de leite caracteriza-se pelo uso, em larga escala, de bovinos mestiços, originados do cruzamento de raças européias e zebuínas, com ampla variedade de graus de sangue. Certamente, tal fato ocorre em razão das condições bioclimáticas do Brasil, onde a criação de raças européias mostra-se muito exigente quanto ao manejo de criação. Entretanto, Dias Gonzales (1991) registrou diferença no desempenho reprodutivo dos animais com diferentes graus de sangue, em aqueles que apresentaram maior fração genética Bos taurus indicus demonstraram maior eficiência na utilização dos alimentos, maior adaptabilidade às condições adversas e tendência a apresentar melhores índices reprodutivos em condições tropicais. Contudo, essas observações mostram-se conflitantes com as de outros estudos, em que animais com maior fração genética Bos taurus taurus demonstraram maior eficiência reprodutiva.

Segundo Dobson \& Kamonpatana (1986), Lamonthe-Zavaleta et al. (1991) e Lobato (1992), fêmeas taurinas apresentaram menor número de serviços ou intervalo parto-concepção que as zebuínas. Teodoro (1991) afirmou que, dependendo do manejo adotado, animais mestiços Bos taurus taurus x Bos taurus indicus podem apresentar resultados de produção e reprodução iguais ou superiores aos animais taurinos.

Diversos estudos vêm sendo realizados com o intuito de esclarecer a fisiologia reprodutiva dos animais taurinos e zebuínos leiteiros e seus cruzamentos (Sirois \& Fortune, 1988; Ginther et al., 1989; Figueiredo et al., 1997; Oliveira, 1997; D'avila de Almeida, 1997; Gambini et al., 1998; Borges, 1999). Entre eles, o da dinâmica folicular tem contribuído no conhecimento da fisiologia ovariana.

Segundo Mcmillan \& Thatcher(1991) e Figueiredo et al. (1997), conhecendo a dinâmica folicular e a fisiologia do corpo lúteo, pode-se aumentar a eficiência reprodutiva com a utilização de farmacológicos, principalmente em programas de inseminação artificial ou de transferência de embriões. Fernandes et al. (1998) observaram diferenças nas respostas à sincronização do estro em fêmeas submetidas ao tratamento com $\mathrm{PGF}_{2} \alpha$ entre os dias 5 e 8 e entre os dias 9 e 17 do ciclo estral, com o último grupo apresentando melhores respostas ao tratamento $(71,87$ vs. $57,44 \%)$. De acordo com esses autores, essa diferença se deve, provavelmente, à quantidade de receptores para a $\mathrm{PGF}_{2} \alpha$ nas células luteínicas, como também seria em função do "status" da onda folicular e do estádio de desenvolvimento dos folículos, principalmente do folículo dominante (Kastelic et al., 1990; Adams et al., 1994; Fernandes et al., 1998).

O crescimento folicular é um fenômeno cíclico e depende da fase do ciclo estral (Pierson \& Ginther, 1988). De acordo com Figueiredo et al. (1997), ocorrem variações no número de ondas foliculares entre os animais de uma mesma raça e num mesmo animal, podendo ocorrer uma, duas, três ou, em menor freqüência, quatro ondas foliculares. Tais variações podem ocorrer em função de vários fatores, como dieta, manejo, produção de leite, período de lactação e pós-parto imediato (Ginther et al., 1996).

Os bovinos apresentam normalmente duas (Pierson \& Ginther, 1988; Oliveira, 1997) ou três (Sirois \& Fortune, 1988; Borges, 1999) ondas foliculares por ciclo. De acordo com Fortune et al. (1988), Ginther et al. (1989) e Taylor \& Rajamahendran (1991), o número de ondas pode ser influenciado pela duração da fase luteínica e, dessa forma, o tratamento com progestágenos exógenos pode aumentar o número de ondas foliculares e a duração do ciclo estral.

O crescimento folicular ocorre de forma contínua, variando de intensidade em função do estádio de desenvolvimento do folículo e do ciclo estral, pois o aumento na taxa de mitose das células do epitélio folicular, assim como o desenvolvimento do antro, são mais acelerados no final do ciclo estral (Lussier et al., 1987).

O folículo dominante de cada onda é capaz de inibir o crescimento e induzir a atresia dos demais folículos em desenvolvimento, além de bloquear o surgimento de uma nova onda folicular (Pierson \& Ginther, 1988; Sávio et al., 1988; Assey et al., 1993). Essa condição foi também observada por Bungartz \& Niemann (1993), que obtiveram maior número de embriões totais e transferíveis em vacas que foram superovuladas, quando não apresentavam um folículo dominante. A inibição do folículo dominante realizase de forma passiva pela redução da concentração do hormônio folículo estimulante (FSH) e de forma ativa, reduzindo o número de receptores ao hormônio nos folículos subordinados. Dessa forma, os hormônios estradiol e inibina, produzidos pelas células da granulosa do folículo dominante, reduzem a liberação de FSH a concentrações muito baixas, insuficientes para manter o desenvolvimento dos folículos subordinados, porém suficientes para manter o crescimento 
do folículo dominante. Segundo Fortune (1994), o folículo dominante consegue manter seu crescimento em função do aumento do número de receptores para gonadotrofinas e aumento do aporte sangüíneo ao folículo.

O presente estudo teve como objetivo estudar a dinâmica folicular e a duração do ciclo estral em alguns grupamentos genéticos, verificando se a fisiologia reprodutiva desses animais é influenciada pela composição genética (grau de sangue) Holandês x Zebu.

\section{Material e Métodos}

O experimento foi realizado no Setor de Bovinocultura do Departamento de Zootecnia da Universidade Federal de Viçosa, em Viçosa, Minas Gerais, no período de agosto a dezembro de 1998.

Foram utilizadas 25 vacas, distribuídas de acordo com a composição genética em cinco grupamentos, sendo: grupo $1=$ vacas $1 / 2$ Holandês $\mathrm{x} Z \mathrm{Zebu}$, grupo 2 $=3 / 4$ Holandês $\mathrm{x}$ Zebu, grupo $3=15 / 16$ Holandês $\mathrm{x}$ Zebu, grupo $4=$ Holandês-PC; e grupo $5=7 / 8$ Holandês x Zebu.

Os animais foram mantidos em semi-estabulação, com suplementação de volumoso fornecida duas vezes ao dia, utilizando-se capim-elefante (P. purpureum) ou associação capim/cana-de-açúcar. $\mathrm{O}$ alimento concentrado foi misturado ao volumoso na proporção de $1 \mathrm{~kg}$ de concentrado (22\% de PB) para cada $3 \mathrm{~kg}$ de leite produzido, com sal mineral e água fornecidos ad libitum.

As observações visuais das manifestações de estro foram realizadas duas vezes ao dia, durante uma hora, das 7 às $8 \mathrm{~h}$ e das 17 às $18 \mathrm{~h}$, com o auxílio de rufiões.

A dinâmica folicular foi avaliada por exames ultra-sonográficos realizados diariamente, com o auxílio de um aparelho de ultra-som (Pie Medical, mod.Vet 200) acoplado ao transdutor de 5,0/7,5 $\mathrm{MHz}$. As mensurações dos folículos em crescimento foram realizadas do dia do estro até à manifestação do estro subseqüente, durante dois ciclos estrais consecutivos. Foram registrados os diâmetros máximos do $1^{\circ}$ e do $2^{\circ}$ maior folículo por onda folicular nos ovários direito e esquerdo, número de ondas foliculares por ciclo estral, período de surgimento e duração das ondas foliculares. A área do corpo lúteo foi mensurada a partir do dia em que foi observado pelo exame ultra-sonográfico até a mani- festação do estro subseqüente.

Amostras de sangue de todos os animais foram coletadas, durante dois ciclos estrais consecutivos, por punção da veia ou artéria coccígea, em frasco contendo anticoagulante (heparina). As coletas foram realizadas duas vezes por semana, com início no dia 0 (estro) ou no $1^{\circ}$ dia pós-estro. Imediatamente após a coleta, as amostras de sangue foram centrifugadas a $3.000 \mathrm{rpm}$ durante 10 minutos e os plasmas obtidos, identificados quanto ao animal e à data da coleta, acondicionados em tubos tipo eppendorf e estocados em "freezer" a $-18^{\circ} \mathrm{C}$ até o momento das dosagens.

As concentrações de progesterona foram analisadas por radioimunoensaio (RIA) com utilização de "Kits" comerciais (ICN PHARMACEUTICALS, INC), no Laboratório de Biofísica do ICB - UFMG, em aparelho cintilador gama Cobra II, marca Apackard. O coeficiente de variação entre e dentro de análise foi de 0,09 e a sensibilidade do teste, de $0,01 \mathrm{ng}$ de progesterona.

Utilizou-se um delineamento inteiramente casualizado com cinco tratamentos (grupamentos genéticos) e cinco repetições (número de animais por grupamentos genéticos). Os dados foram analisados em programa estatístico - SAEG (Universidade Federal de Viçosa, 1998), utilizando-se a estatística descritiva, para obtenção de médias e desvios-padrão para todas as variáveis estudadas; análise de variância e teste $\mathrm{F}$, com probabilidade de $5 \%$, tendo como variável independente o grupamento genético e, como variáveis dependentes, as demais variáveis estudadas. Quando foi verificado efeito pelo teste $F$, realizou-se a comparação de médias pelos testes de NewmanKeuls e Tukey com probabilidade de 5\%. Foram empregadas análises por regressões lineares e quadráticas, tendo como variável dependente a concentração de progesterona e, como variável independente, o dia de coleta. Correlações simples de Pearson foram estabelecidas entre todas as variáveis estudadas.

\section{Resultados e Discussão}

Os valores médios dos períodos interovulatórios observados nos dois ciclos estrais consecutivos são apresentados na Tabela 1. Não se observou diferença $(\mathrm{P}>0,05)$ entre os grupamentos genéticos quanto à duração do ciclo estral de duas ou três ondas

\section{R. Bras. Zootec., v.31, n.2, p.627-634, 2002}


foliculares. Contudo, valores maiores $(\mathrm{P}<0,05)$ foram verificados nos ciclos estrais apresentando três ondas de crescimento folicular $(25,33 \pm 4,37 v s$. $16,0 \pm 3,6$ e $21,83 \pm 1,77$ dias em padrões de três, uma e duas ondas foliculares, respectivamente), corroborando as observações de Taylor \& Rajamahendran (1991). Com relação aos ciclos estrais com duas ondas foliculares, valores semelhantes foram registrados por Sirois \& Fortune (1988), Rhodes et al. (1995) e Hamilton et al. (1995), porém períodos menores foram encontrados por Taylor \& Rajamahendran (1991), que observaram valores de $13,4 \pm 6,2$ e $17,6 \pm 5,0$ dias nos dois ciclos estrais consecutivos; e por Oliveira (1997), em animais imediatamente no pós-parto ( 15 e 17,2 dias nos dois ciclos estrais consecutivos).

Considerando a duração do ciclo de apenas uma onda folicular (16,0 $\pm 3,6$ dias), os valores observados indicaram números absolutos superiores aos registrados por Oliveira (1997), que computou valores de $11,8 \pm 1,9$ dias de duração dos ciclos. Ressalta-se, ainda, que a freqüência elevada de ciclos de apenas uma onda folicular registrada pela referida autora se deve ao fato de que os animais se encontravam no período pós-parto imediato. Tais observações corroboram os estudos de Roche et al. (1992). A duração de ciclos estrais com três ondas foliculares foi similar à observada por Ginther et al. (1989), Taylor \& Rajamahendran (1991) e Borges (1999), porém superior ao registrado por Gambini et al. (1998).

Tabela 1 - Período interovulatório (dias) em dois ciclos estrais consecutivos em um rebanho leiteiro com diferentes graus de sangue Holandês $x$ Zebu, criado em regime semi-extensivo

Table 1 - Interovulatory period (days) in two consecutive estrous cycles in a dairy herd with different crossbreeding Holstein $x$ Zebu level, raised in semi-extensive condition

\begin{tabular}{lccc}
\hline Grau de sangue & Ciclo 1 & Ciclo 2 & Total \\
\hline Holandês-PC & $22,40 \pm 1,52$ & $23,60 \pm 5,86$ & $23,00 \pm 4,08$ \\
$1 / 2$ Hol x Zebu & $20,00 \pm 4,18$ & $20,60 \pm 3,36$ & $20,30 \pm 3,59$ \\
$3 / 4$ Hol x Zebu & $23,20 \pm 1,09$ & $21,00 \pm 1,58$ & $22,10 \pm 1,73$ \\
$7 / 8$ Hol x Zebu & $22,20 \pm 2,05$ & $21,04 \pm 2,97$ & $21,80 \pm 2,44$ \\
$15 / 16$ Hol x Zebu & $22,60 \pm 1,95$ & $22,20 \pm 1,64$ & $22,40 \pm 1,71$ \\
Geral & $22,08 \pm 2,46$ & $21,76 \pm 3,33$ & $21,94 \pm 2,90$ \\
\hline
\end{tabular}

As médias entre os diferentes graus de sangue não foram diferentes $(P>0,05)$.

The average values were similar among the different crossbreeding levels (P>.05).

\footnotetext{
R. Bras. Zootec., v.31, n.2, p.627-634, 2002

Considerando os animais como um único grupo, observou-se a predominância de duas ondas foliculares $(39 / 78 \%)$, sendo verificada também a presença de uma $(3 / 6 \%)$ e três $(8 / 16 \%)$ ondas foliculares, enquanto ciclos com quatro ou mais ondas foliculares não foram constatados neste estudo. Observações semelhantes foram feitas por Taylor \& Rajamahendran (1991), Rhodes et al. (1995) e Oliveira (1997), porém diferiram das observações de Sirois \& Fortune (1988) e Ginther et al. (1989), que registraram a predominância de três ondas foliculares. As variações no número de ondas foliculares, segundo Fortune et al. (1988) e Fortune (1994), provavelmente estejam associadas à duração do ciclo estral e ao tempo de vida útil do corpo lúteo, o que foi também observado neste estudo.

Quando analisado por grupamento genético, somente os animais da raça Holandês e mestiços $1 / 2$ e $3 / 4$ Holandês x Zebu apresentaram ciclos estrais com três ondas de crescimento folicular, enquanto dois animais $1 / 2$ Holandês $x$ Zebu e uma vaca 7/8 Holandês $x$ Zebu exibiram somente uma onda de crescimento folicular durante o ciclo estral. Não se observou um padrão fixo em um mesmo animal no número de ondas foliculares por ciclo estral, ocorrendo a apresentação de uma/duas, três/uma ou duas/três ondas foliculares no primeiro e segundo ciclos estrais consecutivos. Tais observações foram similares às de Figueiredo et al. (1997), que verificaram variação no número de ondas foliculares entre os animais de uma mesma raça, num mesmo animal e de um ciclo para outro ciclo estral.

Em razão do número de ondas foliculares por ciclo estral, a duração das ondas foliculares mostrouse diferente entre os ciclos respectivos, exceto a $1^{\text {a }}$ onda folicular dos ciclos estrais com duas e três ondas foliculares, que foram semelhantes. Tais observações corroboram os registros de Borges (1999).

A duração da $1^{\mathrm{a}}$ e $2^{\mathrm{a}}$ ondas foliculares em ciclos estrais com duas ondas foliculares foi de $11,15 \pm 1,65 \mathrm{e}$ $12,07 \pm 2,32$ dias, respectivamente, sendo os valores da $1^{\mathrm{a}}$ onda similares aos relatados por Ginther et al. (1989), Rhodes et al. (1995) e Oliveira (1997), porém inferiores aos descritos por Sávio et al. (1988), Gambini et al. (1998) e Borges (1999). A duração da $2^{a}$ onda folicular nos ciclos estrais com duas ondas de crescimento mostrou-se próxima aos períodos relatados por Figueiredo et al. (1997), Oliveira (1997) e Borges (1999).

A duração das ondas foliculares nos ciclos com três ondas foliculares foram de $11,00 \pm 3,69 ; 7,17 \pm 1,60$; 
e $8,5 \pm 1,52$ dias na $1^{\mathrm{a}}, 2^{\mathrm{a}}$ e $3^{\mathrm{a}}$ ondas de crescimento, respectivamente. Os valores registrados na $1^{\mathrm{a}}$ onda foram próximos dos relatados por Figueiredo et al. (1997) em animais da raça Nelore, porém divergiram das observações realizadas por Gambini et al. (1998), que verificaram valores de 14,4 dias, e Borges (1999), que encontrou duração de 15,86 dias. Neste estudo, observou-se duração semelhante entre a $2^{\mathrm{a}}$ e $3^{\mathrm{a}}$ ondas foliculares, resultados esses divergentes dos relatados por Ginther et al. (1989), Figueiredo et al. (1997) e Borges (1999), que verificaram menor duração na $3^{\mathrm{a}}$ onda folicular, provavelmente ocorreu do seu surgimento próximo ao início da luteólise e da maior síntese e liberação de hormônio luteinizante, maior número de receptores das células foliculares e maior responsividade folicular ao hormônio hipofisário, resultando em menor tempo para crescimento, maturação e ovulação do folículo. A curta duração da $2^{\mathrm{a}}$ onda folicular provavelmente ocorreu em razão das maiores concentrações plasmáticas de progesterona $\left(10,3\right.$ e $7,19 \mathrm{ng} / \mathrm{mL}$ no $1^{\circ}$ e $2^{\circ}$ ciclos estrais consecutivos) por ocasião do surgimento da onda folicular, com maior feedback negativo da progesterona sobre produção de gonadotrofinas. Porém, quando ocorreram apenas duas ondas foliculares por ciclo estral, não se observou diferença entre as durações da $1^{\mathrm{a}}$ e $2^{\mathrm{a}}$ ondas foliculares.

Nos ciclos com apenas uma onda folicular, o surgimento da onda ocorreu no dia $0,67 \pm 1,15$ do ciclo estral, enquanto com duas ondas foliculares a detecção da $1^{\mathrm{a}}$ e $2^{\mathrm{a}}$ ondas ocorreu nos dias $0 \pm 0$ e $9,85 \pm 1,74$ do ciclo estral, resultados esses semelhantes aos relatados por Ginther et al. (1989), Taylor \& Rajamahendran (1991), Hamilton et al. (1995) e Figueiredo et al. (1997). Nos ciclos com três ondas foliculares, o surgimento da $1^{\mathrm{a}}, 2^{\mathrm{a}}$ e $3^{\mathrm{a}}$ ondas de crescimento ocorreu nos dias $0 \pm 0 ; 10,33 \pm 3,98$; e 16,83 $\pm 4,66$ do ciclo estral, respectivamente, sendo semelhantes aos relatados por Kastelic (1994) e Borges (1999).

O maior diâmetro do folículo dominante, em ciclos de uma onda, foi observado no dia 16,0 $\pm 0,66$. Nos ciclos com duas ondas foliculares, o maior diâmetro do $1^{\circ}$ e $2^{\circ}$ folículos dominantes ocorreu nos dias $7,27 \pm 1,62$ e $21,83 \pm 1,77$, respectivamente, enquanto em ciclos com três ondas foliculares o $1^{\circ}, 2^{\circ}$ e $3^{\circ}$ folículos dominantes alcançaram o diâmetro máximo nos dias 7,33 $\pm 2,80 ; 14,0 \pm 4,69 ;$ e 25,33 $\pm 4,37$ do ciclo estral, respectivamente. Observou-se que o folículo ovulatório sempre apresentou maior diâmetro em relação aos demais folículos da onda folicular, porém não diferiu dos demais folículos dominantes das ondas foliculares do ciclo estral, mesmo quando ocorreram três ondas foliculares. Tais observações não corroboram as de Sirois \& Fortune (1988), Gambini et al. (1998) e Borges (1999), que verificaram o maior diâmetro do folículo ovulatório em relação aos demais folículos dominantes do ciclo.

Os diâmetros médios dos dois maiores folículos encontrados no ovário direito foram: $1,52 \pm 0,39 \mathrm{e}$ $1,40 \pm 0,46 ; 1,63 \pm 0,35$ e $1,44 \pm 0,39$; e $1,97 \pm 0,59$ e $1,77 \pm 0,46 \mathrm{~cm}$, na primeira, segunda e terceira ondas foliculares, respectivamente. Quanto ao ovário esquerdo, os diâmetros médios dos dois maiores folículos encontrados foram de: $0,98 \pm 0,24$ e $0,89 \pm 0,16$; $1,03 \pm 0,24$ e $0,98 \pm 0,24$; e $0,95 \pm 0,19$ e $0,92 \pm 0,17 \mathrm{~cm}$, na primeira, segunda e terceira ondas foliculares, respectivamente. Em todas as mensurações efetuadas, os valores mostraram-se superiores aos registros de D'avila de Almeida (1997) em animais das raças Gir, Holandês e mestiços Holandês x Gir.

O valor médio do diâmetro máximo do folículo dominante foi de $1,87 \pm 0,66 \mathrm{~cm}$ no folículo dominante do ciclo de uma onda folicular. Nos ciclos com duas ondas, foram registrados valores de $1,75 \pm 0,33 \mathrm{e}$ $1,81 \pm 0,24 \mathrm{~cm} \mathrm{no} 1^{\circ}$ e $2^{\circ}$ folículos dominantes, respectivamente. Nos ciclos com três ondas foliculares, os diâmetros foram de $1,65 \pm 0,16 ; 1,72 \pm 0,21 ; \mathrm{e} 1,82 \pm 0,53 \mathrm{~cm}$ no $1^{\circ}, 2^{\circ}$ e $3^{\circ}$ folículos dominantes, respectivamente. Os animais mestiços apresentaram valores de diâmetro folicular em ciclos com duas ou três ondas foliculares semelhantes aos dos animais da subespécie Bos taurus taurus, sendo, portanto, superiores aos dos animais da subespécie Bos taurus indicus, corroborando, assim, as observações de Gambini et al. (1998). Contudo, com relação à variação no número de ondas, a dinâmica folicular mostrou-se semelhante.

Neste estudo, não se verificou diferença entre os grupamentos genéticos com relação à produção leiteira e dias de lactação ( $\mathrm{P}>0,05)$, sendo predominantes fêmeas na primeira lactação, com algumas fêmeas na segunda, terceira e quarta lactações distribuídas aleatoriamente entre os grupamentos genéticos. Nas fêmeas Holandês-PC, as médias encontradas foram de $5.331,1 \pm 1.154,9$ litros e $345,5 \pm 55,9$ dias de lactação; no grupo de fêmeas $1 / 2$ sangue Holandês $x$ Zebu, de 2.779,6 $\pm 1.321,0$ litros e 236,5 $\pm 62,4$ dias; no grupo de fêmeas 3/4 Holandês x Zebu, de $4.180,8 \pm 775,6$ litros e $310,4 \pm 50,6$ dias; no grupo de

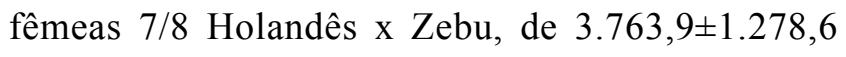
litros e $274,0 \pm 55,2$ dias; e no grupo de fêmeas $15 / 16$ 
Holandês x Zebu, de 3.335,8 $\pm 2364,5$ litros e $266,0 \pm 110,3$ dias de lactação. Nesse rebanho estudado, não se registrou relação da produção de leite com os grupamentos genéticos $(\mathrm{P}>0,05)$, indicando baixa variação genética entre os animais na produção leiteira. Com relação ao escore corporal, todos os animais apresentaram escore entre 3,0 e 3,5 (escala de 1-5) no período experimental, de modo que não se registrou efeito desse parâmetro sobre as características de dinâmica folicular.

Com relação à atividade gonadal, observou-se maior atividade do ovário direito $(31 / 62 \%)$ com relação ao ovário esquerdo (19/38\%), o que está de acordo com os relatos de Pierson \& Ginther (1987; 1988) e D’avila de Almeida (1997). Contudo, não ocorreu no mesmo animal um único padrão, detectando-se a ovulação em gônadas diferentes entre os dois ciclos estudados. Entre os 50 ciclos estrais estudados, em apenas seis ciclos (12\%) não ocorreu crescimento do folículo ovulatório na gônada que apresentava o corpo lúteo, indicando que o corpo lúteo pode estar relacionado à modulação da onda folicular e do folículo dominante.

Os valores médios da área do corpo lúteo e da dosagem de progesterona por grupamento genético no dia de maior concentração são apresentados na Tabela 2. Não se observou diferença $(P>0,05)$ entre as concentrações plasmáticas de progesterona e área do corpo lúteo entre os ciclos estrais e grupamentos genéticos.

Os valores máximos observados neste estudo foram inferiores aos relatados por D' avila de Almeida (1997), embora o dia de maior concentração plasmática de progesterona tenha sido semelhante. Entretanto, mostraram-se próximos aos valores re- portados por Adeyemo \& Heath (1980), Vaca et al. (1983) e Rosa et al. (1988), os quais observaram valores máximos no dia 15 do ciclo estral. Neste estudo, não se verificou nenhuma tendência das concentrações plasmáticas de progesterona, em número absoluto, em serem menores ou maiores nos animais da raça Holandês ou da raça Gir, resultados contraditórios às observações de Agarwal et al. (1977), Adeyemo \& Heath (1980) e D'avila de Almeida (1997).

Quando consideradas as áreas dos corpos lúteos no primeiro ciclo estral, verificou-se que esta foi inferior $(\mathrm{P}<0,05)$ nos animais mestiços $1 / 2$ e $3 / 4$ Holandês x Zebu, em comparação com os animais Holandês-PC e 7/8 Holandês x Zebu, enquanto os animais 15/16 Holandês $x$ Zebu apresentaram área do corpo lúteo similar $(\mathrm{P}>0,05)$ à dos demais grupamentos. No segundo ciclo estral, os animais $1 / 2$ e 3/4 Holandês x Zebu apresentaram corpos lúteos menores $(\mathrm{P}<0,05)$ que os animais $15 / 16$ e $7 / 8$ Holandês x Zebu e Holandês-PC (Tabela 3). No entanto, não houve diferença da área dos corpos lúteos entre os ciclos estudados. A baixa relação dos níveis de progesterona com a área de corpo lúteo neste estudo $(\mathrm{r}=0,09 ; \mathrm{P}=0,17)$ indica que os animais com maior grau de sangue taurino não apresentaram maiores níveis de progesterona, talvez em razão da maior sensibilidade a fatores como temperatura, manejo, maior período de lactação e produção de leite, que podem afetar a atividade das células luteínicas.

A concentração de progesterona, embora tenha demonstrado interação dos efeitos dos ciclos estrais e grupos genéticos estudados, não diferiu entre os grupamentos genéticos, em ambos os ciclos estrais, pela análise de comparação de médias pelo teste de NewmanKeuls e Tukey a $5 \%$ de probabilidade (Tabela 3 ).

Tabela 2 - Área do corpo lúteo $\left(\mathrm{mm}^{2}\right)$ e concentração plasmática de progesterona $(\mathrm{ng} / \mathrm{mL})$ no dia de maior concentração (pico) nos diversos grupamentos genéticos

Table 2 - Corpus luteum area $\left(\mathrm{mm}^{2}\right)$ and plasma progesterone concentration $(\mathrm{ng} / \mathrm{mL})$ in the highest concentration day in the different genetic groups

\begin{tabular}{|c|c|c|c|c|c|c|}
\hline \multirow[t]{2}{*}{ Grupos } & \multicolumn{3}{|c|}{$1^{\circ}$ ciclo } & \multicolumn{3}{|c|}{$2^{\circ}$ ciclo } \\
\hline & Dia & $\mathrm{P}_{4}$ & C. Lúteo & Dia & $\mathrm{P}_{4}$ & C. Lúteo \\
\hline 1/2 Hol x Zebu & $12,2 \pm 2,5$ & $6,78 \pm 4,0$ & $25,6 \pm 12,3$ & $10,8 \pm 5,0$ & $5,50 \pm 2,6$ & $27,2 \pm 13,7$ \\
\hline 3/4 Hol x Zebu & $15,6 \pm 2,7$ & $9,78 \pm 5,7$ & $26,2 \pm 12,0$ & $14,2 \pm 0,8$ & $6,05 \pm 2,4$ & $32,4 \pm 14,2$ \\
\hline 15/16 Hol x Zebu & $13,4 \pm 3,4$ & $6,80 \pm 2,7$ & $37,4 \pm 31,9$ & $11,8 \pm 3,8$ & $5,52 \pm 2,6$ & $46,4 \pm 10,6$ \\
\hline Holandês - PC & $13,8 \pm 4,0$ & $8,73 \pm 6,2$ & $42,4 \pm 11,0$ & $12,8 \pm 3,3$ & $6,20 \pm 1,9$ & $41,4 \pm 6,6$ \\
\hline 7/8 Hol x Zebu & $13,2 \pm 2,6$ & $4,76 \pm 1,2$ & $35,4 \pm 6,1$ & $9,6 \pm 1,7$ & $4,74 \pm 1,1$ & $34,6 \pm 15,0$ \\
\hline Geral & $13,6 \pm 3,0$ & $7,29 \pm 4,3$ & $33,4 \pm 17,0$ & $11,8 \pm 3,4$ & $5,60 \pm 2,0$ & $36,4 \pm 13,3$ \\
\hline
\end{tabular}

*Não houve diferença entre as variáveis estudadas $(P>0,05)$.

* No statistical differences have been found ( $P>$.05). 
A área de corpo lúteo apresentou comportamento quadrático em razão dos dias de mensuração, sendo representada pelas seguintes equações: grupo $1=$ $\left(\mathrm{Y}=9,3899+3,5760 \mathrm{X}-0,1593 \mathrm{X}^{2} \quad \mathrm{P}<0,0001\right.$; $\left.\mathrm{R}^{2}=0,16\right)$; grupo $2=(\mathrm{Y}=1,4858+4,6003 \mathrm{X}-$ $\left.0,1758 X^{2} \mathrm{P}<0,0001 ; \mathrm{R}^{2}=0,15\right)$; grupo $3=(\mathrm{Y}=0,4530$ $\left.+5,3647 \mathrm{X}-0,1967 \mathrm{X}^{2} \mathrm{P}<0,0001 ; \mathrm{R}^{2}=0,13\right)$; grupo $4=$ $\left(\mathrm{Y}=16,9528+3,1949 \mathrm{X}-0,1195 \mathrm{X}^{2} \quad \mathrm{P}<0,0001\right.$; $\left.\mathrm{R}^{2}=0,25\right)$; e grupo $5=(\mathrm{Y}=11,8997+4,5592 \mathrm{X}-$ $0,1807 \mathrm{X}^{2} \quad \mathrm{P}<0,0001 ; \mathrm{R}^{2}=0,22$, em que $\mathrm{Y}=$ área estimada do corpo lúteo e $\mathrm{X}=$ dias de mensuração).

As concentrações plasmáticas de progesterona no primeiro ciclo, não se considerando os grupamentos genéticos, apresentaram comportamento quadrático, em função dos dias de coleta, sendo a equação representada por $\mathrm{Y}=-0,9399+1,0999 \mathrm{X}-0,0453 \mathrm{X}^{2} \mathrm{P}<0,0001$; $\mathrm{R}^{2}=0,22$, em que $\mathrm{Y}=$ progesterona estimada e $\mathrm{X}=$ dia de coleta. No segundo ciclo, essas concentrações tiveram o mesmo comportamento quadrático $(\mathrm{Y}=-0,22+$ $0,5979 \mathrm{X}-0,0241 \mathrm{X}^{2} \mathrm{P}<0,0001 ; \mathrm{R}^{2}=0,23$, sendo $\mathrm{Y}=$ progesterona estimada e $\mathrm{X}=$ dia de coleta).

Quando considerados os grupamentos genéticos, os valores de progesterona no primeiro ciclo apresentaram comportamento quadrático da progesterona em função dos dias de coleta, sendo as melhores equações representadas como se segue:

grupo $1=\left(\mathrm{Y}=-1,1289+1,0339 \mathrm{X}-0,0448 \mathrm{X}^{2}\right.$ $\left.\mathrm{P}<0,0008 ; \mathrm{R}^{2}=0,36\right)$; grupo $2=(\mathrm{Y}=-2,1994+1,239 \mathrm{X}$ $\left.-0,0519 \mathrm{X}^{2} \quad \mathrm{P}<0,0001 ; \mathrm{R}^{2}=0,53\right)$; grupo $3=$ $\left(\mathrm{Y}=-2,0574+1,1296 \mathrm{X}-0,0486 \mathrm{X}^{2} \quad \mathrm{P}<0,0003\right.$; $\left.\mathrm{R}^{2}=0,43\right)$; grupo $4=(\mathrm{Y}=-1,5280+0,3711 \mathrm{X}-$ $\left.0,0141 \mathrm{X}^{2} \mathrm{P}<0,02 ; \mathrm{R}^{2}=0,22\right)$; e grupo $5=(\mathrm{Y}=-0,4482$ $+0,6753 \mathrm{X}-0,02935 \mathrm{X}^{2} \mathrm{P}<0,0001 ; \mathrm{R}^{2}=0,41$, sendo $\mathrm{Y}=$ progesterona estimada e $\mathrm{X}=$ dia de coleta).

Considerando os valores de progesterona no segundo ciclo e entre os grupamentos genéticos, também se observou comportamento quadrático da progesterona em função dos dias de coleta, sendo as melhores equações representadas como se segue: grupo $1=\left(\mathrm{Y}=-1,31349+1,2574 \mathrm{X}-0,0593 \mathrm{X}^{2}\right.$ $\left.\mathrm{P}<0,0003 ; \mathrm{R}^{2}=0,32\right)$; grupo $2=(\mathrm{Y}=-0,6103+1,3130 \mathrm{X}$ - 0,0533X $\left.\quad \mathrm{P}<0,007 ; \mathrm{R}^{2}=0,24\right)$; grupo $3=$ $\left(\mathrm{Y}=-1,4651+1,0839 \mathrm{X}-0,0434 \mathrm{X}^{2} \quad \mathrm{P}<0,0001\right.$; $\left.\mathrm{R}^{2}=0,47\right)$; grupo $4=(\mathrm{Y}=-1,1829+1,4166 \mathrm{X}-$ $\left.0,0580 \mathrm{X}^{2} \quad \mathrm{P}<0,006 ; \mathrm{R}^{2}=0,22\right) ;$ e grupo $5=$ $\left(\mathrm{Y}=-1,0697+0,8002 \mathrm{X}-0,03280 \mathrm{X}^{2} \quad \mathrm{P}<0,0004\right.$; $\mathrm{R}^{2}=0,44$, sendo $\mathrm{Y}=$ progesterona estimada $\mathrm{X}=$ dia de coleta).

Tabela 3 - Valores médios e desvio-padrão da área do corpo lúteo e concentração plasmática de progesterona nos grupos estudados em dois ciclos consecutivos

Table 3 - Corpus luteum and plasma progesterone concentration average values and standard deviation for the studied groups in two consecutive estrous cycle

\begin{tabular}{|c|c|c|c|c|}
\hline \multirow[t]{3}{*}{ Grupos } & \multirow{2}{*}{\multicolumn{2}{|c|}{$\begin{array}{c}\text { Progesterona }(\mathrm{ng} / \mathrm{mL}) \\
\text { Progesterone }\end{array}$}} & \multirow{2}{*}{\multicolumn{2}{|c|}{$\begin{array}{c}\text { Área Corpo lúteo }\left(\mathrm{mm}^{2}\right) \\
\text { Corpus luteum }\end{array}$}} \\
\hline & & & & \\
\hline & $1^{\circ} \mathrm{Ciclo}$ & $2^{\circ}$ Ciclo & $1^{\circ}$ Ciclo & $2^{\circ}$ Ciclo \\
\hline 1/2 Hol x Zebu & $3,28 \pm 3,44^{\mathrm{a}}$ & $2,94 \pm 2,93^{\mathrm{a}}$ & $23,54 \pm 11,63^{b}$ & $25,48 \pm 12,15^{b}$ \\
\hline 3/4 Hol x Zebu & $5,02 \pm 4,62^{\mathrm{a}}$ & $3,25 \pm 2,48^{\mathrm{a}}$ & $26,17 \pm 14,28^{\mathrm{b}}$ & $26,34 \pm 13,68^{b}$ \\
\hline 15/16 Hol x Zebu & $3,21 \pm 2,88^{\mathrm{a}}$ & $2,72 \pm 2,52^{\mathrm{a}}$ & $29,07 \pm 21,61^{\mathrm{ab}}$ & $31,90 \pm 14,52^{\mathrm{a}}$ \\
\hline Holandês - PC & $4,92 \pm 5,06^{\mathrm{a}}$ & $3,09 \pm 2,68^{\mathrm{a}}$ & $32,04 \pm 14,18^{\mathrm{a}}$ & $34,14 \pm 13,30^{\mathrm{a}}$ \\
\hline 7/8 Hol x Zebu & $2,58 \pm 1,90^{\mathrm{a}}$ & $2,15 \pm 1,93^{\mathrm{a}}$ & $34,12 \pm 09,33^{\mathrm{a}}$ & $36,07 \pm 14,33^{\mathrm{a}}$ \\
\hline
\end{tabular}

$a, b$ Letras minúsculas diferentes na mesma coluna diferem pelo teste de Newman-Keuls $(P<0,05)$.

$a, b \quad$ Different small letters in the same collun indicate differences, according to Newman-Keuls test $(P<.05)$.

\section{Conclusões}

Conclui-se que o crescimento folicular não apresentou padrão fixo de número de ondas foliculares nos dois ciclos estrais consecutivos, observados nas avaliações de um mesmo animal ou dentro e entre grupamentos genéticos, sendo registrada amplitude de uma a três ondas foliculares, porém com predominância de duas ondas foliculares.

\section{Agradecimento}

À FAPEMIG, pelo financiamento integral deste estudo, e ao Setor de Bovinocultura do Departamento de Zootecnia da Universidade Federal de Viçosa, em Viçosa, Minas Gerais, por disponibilizar a estrutura física e os animais experimentais. 


\section{Literatura Citada}

ADAMS, G.P.; EVANS, A.C.O.; RAWLINGS, N.C. Follicular waves and circulating gonadotrophins in 8-month-old prepubertal heifers. Journal of Reproduction and Fertility, v.100, p.27-33, 1994.

ADEYEMO, O.; HEATH, E. Plasma progesterone concentration in Bos taurus and Bos indicus heifers. Theriogenology, v.14, p.411-420, 1980.

AGARWAL, S.P.; RAHMAN, S.A.; LAUMAS, K.R. Studies on steroid hormones: progesterone concentrations in the blood serum of Zebu cows during oestrous cycle. Indian Journal of Animal Science, v.47, p.411-421, 1977.

ASSEY, R.J.;HYTTEL, P.; PURWANTARA, B. Oocyte morphology in dominant and subordinate follicles during the first follicular wave in cattle. Theriogenology, v.39, p.183-198, 1993.

BORGES, A. M. Dinâmica folicular e superovulação em novilhas mestiças tratadas com somatotropina bovina (rBST) e efeito da temperatura na qualidade dos embriões. Viçosa, MG: Universidade Federal de Viçosa, 1999. 100p. Dissertação (Mestrado em Zootecnia)-Universidade Federal de Viçosa, 1999.

BUNGARTZ, L.; NIEMANN, H. Effects of a dominant follicle on ovarian responses of dairy cattle following various superovulatory treatment schedules. Theriogenology, v.9, p.198-207, 1993.

D'AVILA DE ALMEIDA, C. Ultra-sonografia ovariana e concentrações de progesterona em novilhas da raça Holandesa, Gir e mestiças Holandês-Gir. Viçosa, MG: Universidade Federal de Viçosa, 1997. 60p. Dissertação (Mestrado em Zootecnia) Universidade Federal de Viçosa, 1997.

DIAS GONZALES, F.H. Efeito da condição corporal de novilhas sobre a fertilidade, perfil metabólico pós-serviço e a sobrevivência embrionária. Viçosa, MG: Universidade Federal de Viçosa, 1991. 160p. Tese (Doutorado em Zootecnia) - Universidade Federal de Viçosa, 1991.

DOBSON, H.; KAMONPATANA, M.A. A review of female cattle reproduction with special reference to a comparison between buffaloes, cows and Zebu. Journal of Reproduction and Fertility, v.77, p.1-36, 1986.

FERNANDES, C.A.C.; FERREIRA, A.M.; SÁ, W.F. et al. Sincronização do estro em bovinos de acordo com a fase do ciclo estral. Revista Brasileira de Ciência Veterinária, v.5, p.39-41, 1998.

FIGUEIREDO, R.A.; BARROS, C.M.; PINHEIRO, O.L. et al. Ovarian follicular dynamics in Nelore breed (Bos taurus indicus) cattle. Theriogenology, v.47, p.1489-1505, 1996.

FORTUNE, J.E. Ovarian follicular growth and development in mammals. Biology of Reproduction, v.50, p.225-232, 1994.

FORTUNE, J.E.; SIROIS, J.; QUIRK, S.M. The growth and differentiation of ovarian follicles during the estrous cycle. Theriogenology, v.29, p.95-109, 1988.

GAMBINI, A. L.G.; MOREIRA, M.B.P.; CASTILHO, C. et al. Desenvolvimento folicular e sincronização da ovulação em vacas da raça Gir. Revista Brasileira de Reprodução Animal, v.22, p.201-210, 1998.

GINTHER, J.O.; KNOPF, L.; KASTELIC, J.P. Temporal association among events in cattle during oestrous cycle with two or three follicular waves. Journal of Reproduction and Fertility, v.87, p.223-230, 1989.

GINTHER, O.J.; WILTBANK, M.C.; FRICKE, P.M. et al. Selection of the dominant follicle in cattle. Biology of Reproduction, v.55, p1187-1194, 1996.

HAMILTON, S.A.; GARVERICK, H.A.; KEISLER, D.H. et al. Characterization of ovarian follicular cysts and associated endocrine profiles in dairy cows. Biology of Reproduction, v.53, p.890-898, 1995.

KASTELIC, J.P. Understanding ovarian follicular development in cattle. Veterinary Medicine, v.6, p.64-71, 1994.

KASTELIC, J.P.; KO, J.C.H.; GINTHER, O.J. Suppression of dominant and subordinate ovarian follicles by a proteinaceous fraction of follicular fluid in heifers. Theriogenology, v.34, p.499-509, 1990.

LAMONTHE-ZAVALETA, C.; FREDRIKSSON, G.; KINDAHL, H. Reproductive performance in Zebu cattle in Mexico. 1. Sexual behavior and seasonal influence on estrous cyclicity. Theriogenology, v.36, p.887-896, 1991.

LOBATO, V. Desempenho reprodutivo e produtivo de vacas mestiças leiteiras, submetidas a dois níveis nutricionais, nos períodos pré e pós-parto. Viçosa, MG: Universidade Federal de Viçosa, 1992. 186p. Dissertação (Mestrado em Zootecnia) - Universidade Federal de Viçosa, 1992.

LUSSIER, J.G.; MATTON, P.; DUFOUR, J.J. Growth rates of follicles in the ovary of cow. Journal of Reproduction and Fertility, v.81, p.301-307, 1987.

McMILLAN, K.L.; THATCHER, W.W. Effects of an agonist of gonadotropin-releasing hormone on ovarian follicles in cattle. Biology of Reproduction, v.45, p.883-889, 1991.

OLIVEIRA, M.M.N.F. Dinâmica folicular ovariana e características reprodutivas de vacas leiteiras no pós-parto após tratamentos com buserelina e cloprostenol. Viçosa, MG: Universidade Federal de Viçosa, 1997. 87p. Dissertação (Mestrado em Zootecnia) - Universidade Federal de Viçosa, 1997.

PIERSON, R.A.; GINTHER, O.J. Follicular populations during the estrous cycle in heifers. I. Influence of day. Animal Reproduction Science, v.14, p.165-176, 1987.

PIERSON, R.A.; GINTHER, O.J. Ultrassonic imaging of the ovaries and uterus in cattle. Theriogenology, v.29, p.21-37, 1988.

ROSA, G.O.; LOBREIRO, J.C.T.; DODE, M.A.N. Desempenho reprodutivo de fêmeas Zebuínas. Zootecnia, v.26, p.219-236, 1988.

RHODES, F.M.; FITZPATRICK, L.A.; ENTWISLE, K.W. et al. Hormone concentrations in the caudal vena cava during the first ovarian follicular wave of the oestrous cycle in heifers. Journal of Reproduction and Fertility, v.104, p.33-39, 1995.

ROCHE, J.F.; CROWE, M.A.; BOLAND, M.P. et al. Postpartum anoestrous in dairy and beef cows. Animal Reproduction Science, v.28, p.371-378, 1992.

SÁVIO, J.D.; KEENAN, L.; BOLAND, M.P. et al. Pattern of growth of dominant follicles during the oestrous cycle in heifers. Journal of Reproduction and Fertilility, v.83, p.663-671, 1988.

SIROIS, J.; FORTUNE, J.E. Ovarian follicular dynamics during the estrous cycle in heifers monitored by real-time ultrasonography. Biology of Reproduction, v.39, p.308-317, 1988.

TAYLOR, C.; RAJAMAHENDRAN, R. Follicular dynamics, corpus luteum growth and regression in lactating dairy cattle. Canadian Journal of Animal Science, v.71, p.61-68, 1991.

TEODORO, R.L. Estudo comparativo de desempenho de vacas mestiças Holandês $x$ Gir e vacas do cruzamento tríplice Jersey ou Suíço versus Holandês x Gir. Viçosa, MG: Universidade Federal de Viçosa, 1991. 156p. Dissertação (Mestrado em Zootecnia) - Universidade Federal de Zootecnia, 1991.

UNIVERSIDADE FEDERAL DE VIÇOSA - UFV. Sistema de análises estatísticas e genéticas - SAEG. Viçosa, MG: Universidade Federal de Viçosa, 1998. 150p.

VACA, L.A.; GALINA, C.; FERNÁNDES-BACA, S. et al. Progesterone levels and relationship with the diagnosis of corpus luteum by retal palpation during the estrous cycle in Zebu cows. Theriogenology, v.20, p.67-77, 1983. 